\title{
Coenzyme A-transferase-independent butyrate re-assimilation in Clostridium acetobutylicum - Evidence from a mathematical model
}

\author{
Thomas Millat $^{1,3}$, Christine Voigt ${ }^{2}$, Holger Janssen ${ }^{2}$, Clare M. Cooksley ${ }^{3}$, Klaus Winzer ${ }^{3}$, \\ Nigel P. Minton ${ }^{3}$, Hubert Bahl ${ }^{2}$, Ralf-Jörg Fischer ${ }^{2}$, and Olaf Wolkenhauer ${ }^{1,4}$ \\ ${ }^{1}$ University of Rostock, Institute of Computer Science, \\ Department of Systems Biology \& Bioinformatics, Ulmenstr. 69, 18057 Rostock, Germany \\ ${ }^{2}$ University of Rostock, Institute of Biological Sciences, \\ Division of Microbiology, A.-Einstein-Str. 3, 18051 Rostock, Germany \\ ${ }^{3}$ University of Nottingham, School of Life Sciences, BBRSC Sustainable Bioenergy Centre, \\ Clostridia Research Group, Nottingham NG7 2RD, UK \\ ${ }^{4}$ Institute for Advanced Study (STIAS), Wallenberg Research Centre at Stellenbosch University, \\ Stellenbosch 7600, South Africa
}

(Dated: July 21, 2014)

\begin{abstract}
The hetero-dimeric CoA-transferase CtfA/B is believed to be crucial for the metabolic transition from acidogenesis to solventogenesis in Clostridium acetobutylicum as part of the industrial-relevant acetone-butanol-ethanol (ABE) fermentation. Here, the enzyme is assumed to mediate re-assimilation of acetate and butyrate during a $\mathrm{pH}$-induced metabolic shift and to faciliate the first step of acetone formation from acetoacetyl-CoA. However, recent investigations using phosphate-limited continuous cultures have questioned this common dogma.

To adress the emerging experimental discrepancies, we investigated the mutant strain Cac-ctfA398s::CT using chemostat cultures. As a consequence of this mutation, the cells are unable to express functional ctfA and are thus lacking CoA-transferase activity. A mathematical model of the $\mathrm{pH}$-induced metabolic shift, which was recently developed for the wild type, is used to analyse the observed behaviour of the mutant strain with a focus on re-assimilation activities for the two produced acids.

Our theoretical analysis reveals that the ctfA mutant still re-assimilates butyrate, but not acetate. Based upon this finding, we conclude that $C$. acetobutylicum possesses a CoA-tranferase-independent butyrate uptake mechanism that is activated by decreasing $\mathrm{pH}$ levels. Furthermore, we observe that butanol formation is not inhibited under our experimental conditions, as suggested by previous batch culture experiments. In concordance with recent batch experiments, acetone formation is abolished in chemostat cultures using the $c t f a$ mutant.
\end{abstract}

Keywords Clostridium acetobutylicum; ctfA mutant; Acid re-assimilation; $\mathrm{pH}$-induced metabolic shift; Mathematical modelling

Author to whom correspondence should be addressed:

Thomas Millat,

Clostridia Research Group,

School of Life Sciences,

University of Nottingham, Nottingham NG7 2RD;

Tel. +44 (0)115 95 15074;

Email: thomas.millat@nottingham.ac.uk 


\section{Introduction}

The acetone-butanol-ethanol (ABE) fermentation carried out by $C$. acetobutylicum comprises two distinct metabolic states that differ in their product formation. During exponential growth acetate and butyrate are produced (acidogenic phase). This type of anaerobic metabolism enables the organism to gain the maximal amount of energy (ATP) per mole glucose using substrate-level phosphorylation (Madigan et al. 2009). During the transition to the stationary phase the metabolism switches to the formation primarily of acetone and butanol (solventogenic phase). During this growth phase, the previously excreted acids are re-assimilated. In a continuous culture under phosphate limitation, changes of the external $\mathrm{pH}$ induce the transition between the two metabolic states (Bahl et al. 1982; Fischer et al. 2006). During both phases, ethanol is produced in minor amounts.

The re-assimilation of acetate and butyrate is particularly obvious in batch culture experiments (Jones and Woods 1986). Several biochemical studies have identified a hetero-dimeric CoA-transferase, $\mathrm{CtfA} / \mathrm{B}$, to play a key role in re-assimilation of acids and their conversion to the respective CoA-derivatives (Hartmanis et al. 1984a; Wiesenborn et al. 1989a) and it is, therefore, believed that this enzyme has a fundamentally different role in C. acetobutylicum compared to other bacteria (Wiesenborn et al. 1989a). In addition, it facilitates the first step in the formation of acetone (Andersch et al. 1983; Hartmanis and Gatenbeck 1984). This view is supported by several experimental studies which had observed an increased transcription of the encoding genes, ctfA and ctfB, which are part of the sol operon (Grimmler et al. 2011; Jones et al. 2008), and an increased intracellular concentration of the protein during the $\mathrm{pH}$-induced shift and during solventogenesis (Janssen et al. 2010; Mao et al. 2010). However, recent experimental evidence indicates that an alternative re-assimilation mechanism for butyrate could exist which is independent of the CoA-transferase (Desai et al. 1999; Lehmann et al. 2012a) and may rely on a pH-dependent reverse activity of the butyrate forming enzymes (Desai et al. 1999; Lehmann et al. 2012b). However, this activity would not be consistent with the results reported in (Hartmanis et al. 1984), where the authors concluded from ${ }^{13} \mathrm{C}$ NMR studies, enzyme assays, and thermodynamic considerations that a CoA-transferase-mediated mechanism is more likely responsible for acid re-assimilation than a reverse action of acetate and butyrate formation pathways, acyl-CoA synthase reactions, or generation of acetyl and butyryl phosphate followed by their direct reduction to the corresponding aldehyde. Additionally, our recent theoretical analysis of the product formation rate, using the wild type ATCC 824 (COSMIC strain, see Materials and Methods), suggests that the re-assimilation of acetate is less active than that of butyrate in phosphate-limited continuous cultures (Millat et al. 2013b).

To further elucidate the role of the CoA-transferase in the re-assimilation of acids during the $\mathrm{pH}$-induced metabolic switch, we used a recently developed standard operation procedure (SOP) for the anaerobic growth of $C$. acetobutylicum in a phosphate-limited chemostat (Fiedler et al. 2008; Janssen et al. 2010). Growing on glucose as the sole source of carbon and energy, first a steady state under acidogenic conditions is established, before the $\mathrm{pH}$-induced metabolic switch is induced by allowing the external $\mathrm{pH}$ to drop (see Section "Material and methods"). In comparison to batch cultures, continuous cultures offer the advantage of generating highly reproducible, reliable, and homogeneous data - a crucial prerequisite for global transcriptomic, proteomic, and metabolomic studies. Furthermore, secondary growth and stress responses of cells growing in a batch might obscure physiological differences (Hoskisson and Hobbs 2005). For our experiments we used the wild type strain ATCC 824 (COSMIC strain) and the group II intron retargeted mutant strain Cac-ctfA398s::CT originating from the COSMIC strain (Cooksley et al. 2012).

To elucidate the processes involved in acid re-assimilation, here we have focussed on the dynamic transition phase from acidogenesis to solventogenesis induced by a changing $\mathrm{pH}$ level in forward-shift experiments using a phosphate-limited chemostat (Fiedler et al. 2008; Janssen et al. 2010). As a consequence of the mutation, the acetoacetate formation and, consecutively, the formation of acetone are abolished. The experimentally measured time courses of the external $\mathrm{pH}$, the optical density $\left(\mathrm{OD}_{600}\right)$, and the fermentation products provide the basis for a theoretical 
investigation of the metabolic switch in the mutant, thereby modifying our two-population model developed for the wild type (Millat et al. 2013b).

The model in Millat et al. (2013b) combines the $\mathrm{pH}$-dependent growth of an acidogenic and a solventogenic population with a $\mathrm{pH}$-dependent metabolic model of clostridial ABE fermentation. As a consequence, it distinguishes the two involved processes, acid re-assimilation and drop of population size that determine the decline of the acids after the initiation of the $\mathrm{pH}$-shift. Due to the knock-out of the ctfA gene, the decrease of the acid concentrations should be completely dictated by the population growth and the wash-out from the fermenter, if no alternative mechanisms for re-assimilation exist. In accordance with our expectations, the observed acetate concentrations prove that this acid is not assimilated during the metabolic shift. Contrary to current opinion, our simulation suggests an alternative mechanism for butyrate re-assimilation.

In this study we have compared experimental data to the simulated mathematical model of the ABE network, focussing on the initial transition phase between acidogenesis and solventogenesis, which is characterized by a drop in optical density and the acids acetate and butyrate. The results and their implications are discussed with respect to $\mathrm{ABE}$ fermentation in C. acetobutylicum.

\section{Materials and methods}

Organism and growth conditions. The CoA-transferase defective strain C.acetobutylicum Cac-ctfA398s::CT was recently constructed and analysed as described in Cooksley et al. (2012). For the purpose of systematic comparison between the mutant and the wild type, the experiments were conducted using the same experimental setup and standard operating procedure (SOP) as for the type strain C. acetobutylicum ATCC 824 (COSMIC strain, Rostock lab collection) (Janssen et al. 2010). The additional specification 'COSMIC strain' emphasizes that all works in the trans-national COSMIC1 and COSMIC2 systems biology projects used the same C. acetobutylicum ATCC 824 strain derivative originating from the Rostock culture collection to minimize adverse effects caused by inherent genetic variability. The anaerobic chemostat cultivations were conducted at $37{ }^{\circ} \mathrm{C}$ after a standard operating procedure (SOP) developed by Janssen et al. (2010). In brief, pre-cultures were always freshly inoculated from spore stocks (Fischer et al. 2006) and the phosphate-limited chemostat experiments were performed using a synthetic medium with $0.5 \mathrm{mM} \mathrm{KH} \mathrm{PO}_{4}$ and $4 \%$ (wt/vol) glucose in a BiostatB 1.5-1 fermenter system (Sartorius BBI Systems GmbH, Melsungen, Germany) (Fiedler et al. 2008). The dilution rate (respective generation time) was $\mathrm{D}=0.075 \mathrm{~h}^{-1}$. During the forward-shift experiments, the clostridial population initially grew at an acidogenic $\mathrm{pH}$ level 5.7 for around 5 days. Within this period, an acidogenic steady state was established. Then, the $\mathrm{pH}$ buffering transiently was stopped until the acid producing cells had acidified the external medium to the solventogenic level of $\mathrm{pH} 4.5$. Thereafter, the $\mathrm{pH}$ was kept constant by automated addition of $2 \mathrm{M} \mathrm{KOH}$. Optical density at $600 \mathrm{~nm}\left(\mathrm{OD}_{600}\right)$ was measured by a photometer, and the fermentation products (acetate, butyrate, butanol, acetone, and ethanol) were determined by gas chromatography, respectively (Fischer et al. 2006). The experimental data of three independent forward-shift experiments using the ctfA mutant strain are given in Tables S1-S3 in Online Ressource 1.

\section{Results}

\section{Kinetic modelling of the metabolic shift}

Existing kinetic models of the ABE fermentation in C. acetobutylicum typically consider the wild type of C. acetobutylicum. Papoutsakis developed a stoichiometric model in 1984 (Papoutsakis 1984). Desai at al. (1999) analysed the contribution of acid formation pathways in the metabolism of C. acetobutylicum ATCC 824 by using metabolic flux analysis. Genome-scaled models were applied to investigate the overall flux through the whole cell by several authors (McAnulty et al. 2012; Milne et al. 2011). However, these models lack regulatory and dynamic information. A first kinetic simulation model, to describe the dynamics of ABE fermentation in the related Clostridium saccharoperbutylacetonicum 1-4 during growth in batch culture under condition of glucose 
depletion, was presented in (Shinto et al. 2007). A first model of the $\mathrm{pH}$-induced metabolic shift in C. acetobutylicum in phosphate-limited continuous culture, was published by Haus et al. (2011), which took into account an adaptation of gene expression and proteome composition to the changing external $\mathrm{pH}$. Additionally, the effect of several single mutations on the product formation at acidogenic and solventogenic steady state was investigated. Recently, we developed a model that combines the growth and the network of ABE fermentation of clostridial populations in continuous cultures under phosphate limitation (Millat et al. 2013b). In this model, we proposed that a phenotypic switch governs the $\mathrm{pH}$-induced metabolic switch under these experimental conditions. In particular, the consideration of the population dynamics is important for the investigation presented here, because it separates the effect of changing population sizes from intracellular alterations. In the following section, this mathematical model is introduced briefly, for a more detailed description we refer to (Millat et al. 2013b).

\section{Mathematical model of the ABE fermentation in continuous culture}

In the present manuscript, we apply the method established in Millat et al. (2013b) to investigate the behaviour of the mutant Cac-ctfA398s::CT lacking CoA-transferase activity. Here, we focus on the decrease of acetate and butyrate initiated by the drop of the external $\mathrm{pH}$ level from $\mathrm{pH}=5.7$ to $\mathrm{pH}=4.5$ in phosphate-limited continuous cultures. This procedure is referred to as 'forward-shift' experiment (see also Section "Material and methods").

Our modelling approach considers two subpopulations, an acid-forming phenotype and a solvent-forming phenotype, and their $\mathrm{pH}$-dependent metabolic network. In doing so, we separate the $\mathrm{pH}$-induced metabolic alterations from the changing population growth, which both naturally contribute to the observed drop of acetate and butyrate after the initiation of the $\mathrm{pH}$-shift. According to current dogma, the inactive CoA-transferase in Cac-ctfA398s::CT should result in a decrease of the acid concentrations dominated by the population dynamics of the acid-forming population and the wash-out of the fermentation products from the chemostat.

Recent experimental evidence (Fontaine et al. 2002; Grimmler et al. 2011; Janssen et al. 2010; Janssen et al. 2012 (The last three studies used the COSMIC strain.)) and theoretical investigations (Haus et al. 2011; Millat et al. 2013b) indicate that the pH-induced metabolic shift under our experimental conditions is linked to a rearrangement of transcriptomic, proteomic, and metabolomic composition of the cells. Following our approach demonstrated in Millat et al. (2013b), these experimental findings correspond to an acidogenic and a solventogenic phenotype which are prevalently present either at acidogenic ( $\mathrm{pH}$ above 5.2) or solventogenic conditions ( $\mathrm{pH}$ below 5.1) (Millat et al. 2013a). As a consequence of the $\mathrm{pH}$-shift, the perturbations of the metabolism of the acidogenic population result in a significant inhibition of growth and a consequent dramatic fall in the population size (Grupe and Gottschalk 1992; Janssen et al. 2010; Sauer and Dürre 1995). Simultaneously, a solvent-forming population emerges that eventually establishes a new solventogenic steady state.

The $\mathrm{pH}$-shift is expressed employing an exponential function which is used afterwards to join the three independent forward-shift experiments into a single representation (Appendix 1). Assuming that the optical density $\left(\mathrm{OD}_{600}\right)$ is a measure of the cellular growth, the population's dynamic is described as the sum of an acidogenic $\left(O D^{\mathrm{A}}\right)$ and a solventogenic $\left(O D^{\mathrm{S}}\right)$ population

$$
O D(t)=O D^{\mathrm{A}}(t)+O D^{\mathrm{S}}(t)
$$

prevalently dominating the clostridial population at steady state. During the $\mathrm{pH}$-induced metabolic switch the two populations coexist, see also Appendix 2. In accordance with experimental data, both phenotypes differ in their transcriptomic and proteomic profiles, specific enzyme activities, and thus, in their product formation. As shown in Fig. 1, it is assumed that the acid-forming phenotype produces acetate, butyrate and lesser amounts of ethanol as final liquid products. The solvent-forming phenotype is characterized by the formation of butanol and ethanol, but it also posseses a residual activity of acid formation. No acetone is formed in both phenotypes as a 
consequence of the mutation. Their population sizes during the forward-shift experiments are determined by two different growth functions fitted to the OD data (Appendix 2).

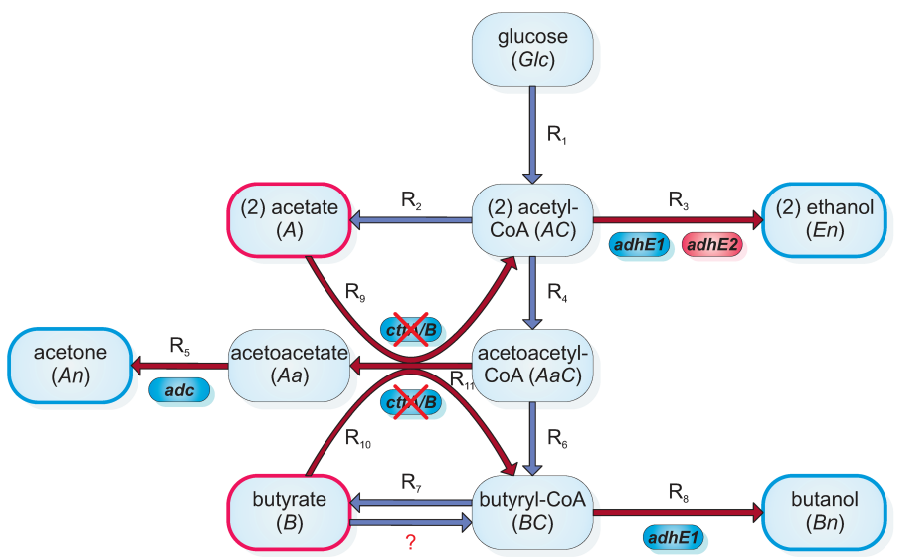

Fig. 1 Simplified metabolic pathway of ABE fermentation in the mutant strain Cac-ctfA398s::CT encoded in the mathematical model (4). During acidogenesis $(\mathrm{pH}<5.2)$, the acids acetate and butyrate are the main products, whereas the solvents acetone and butanol are mainly produced during solventogenesis $(\mathrm{pH}<5.1)$ in continuous culture using phosphate limitation. Ethanol is produced in similar amounts under both conditions. $R_{i}$ denotes the metabolic reaction in the model. Enzymes induced either during solventogenesis (blue) or acidogenesis (red) are highlighted (Grimmler et al. 2011; Janssen et al. 2010). These enzymes are considered with distinct concentrations for both metabolic phases in the model. Red crosses indicate that the CoA transferase gene (ctfA) is interrupted in the mutant strain using ClosTron technology resulting in inhibition of reactions $R_{9}$ and $R_{10}$ (acid re-assimilation), and $R_{11}$ (formation of acetoacetate from acetoacetyl-CoA). Furthermore, a hypothetical reverse mechanism for CoA-transferase-independent butyrate re-assimilation is indicated by a question mark, see also Eq. (5).

The metabolic model, describing the flow through the ABE network, shown in Fig. 1, is composed of eleven rate equations

$$
\frac{d X_{j}}{d t}=\sum_{i} \varepsilon_{i j} R_{i}-D \cdot X_{j}
$$

which describe the changes in metabolite concentrations as a sum over all metabolic reactions $R_{i}$ that contribute to the concentration of metabolites $X_{j}$. The parameter $\varepsilon_{i j}$ is $\varepsilon_{i j}=1$ if $X_{j}$ is produced in the reaction $R_{i}, \varepsilon_{i j}=-1$ if it is consumed in that reaction, or $\varepsilon_{i j}=0$ else. Furthermore, the wash-out of metabolites is considered by a transport term which is the product to the constant dilution rate $D=0.075 \mathrm{~h}^{-1}$ and the metabolite concentration $X_{j}$.

To account for different metabolic activities in the two populations, the rates $R_{i}$ are expressed as a sum over acid- and solvent-forming cells

$$
R_{i}=R_{i}^{\mathrm{A}} \cdot O D^{\mathrm{A}}+R_{i}^{\mathrm{S}} \cdot O D^{\mathrm{S}} .
$$

The corresponding metabolic reactions are defined as

$$
\begin{array}{rll}
R_{1}^{\mathrm{A}}=R_{1}^{\mathrm{S}}=\frac{2 \cdot V_{1} G}{K_{1}+G} & \text { glucose } \rightarrow \text { acetyl-CoA } \\
R_{2}^{\mathrm{A}}=\frac{V_{2} \cdot A C}{K_{2}+A C} & R_{2}^{\mathrm{S}}=\frac{V_{2}}{f_{2}} \frac{A C}{K_{2}+A C} & \text { acetyl-CoA } \rightarrow \text { acetate } \\
R_{3}^{\mathrm{A}}=\alpha_{32} \cdot A C \cdot A d h E 2 & R_{3}^{\mathrm{S}}=\alpha_{31} \cdot A C \cdot A d h E 1 & \text { acetyl-CoA } \rightarrow \text { ethanol } \\
R_{4}^{\mathrm{A}}=R_{4}^{\mathrm{S}}=\frac{V_{4} \cdot A C}{2\left(K_{4}+A C\right)} & \text { acetyl-CoA } \rightarrow \text { acetoacetyl-CoA }
\end{array}
$$




$$
\begin{aligned}
& R_{5}^{\mathrm{A}}=\alpha_{5} \cdot A a \cdot A d c^{\mathrm{A}} \quad R_{5}^{\mathrm{S}}=\alpha_{5} \cdot A a \cdot A d c^{\mathrm{s}} \quad \text { acetoacetate } \rightarrow \text { acetone } \\
& R_{6}^{\mathrm{A}}=R_{6}^{\mathrm{S}}=\frac{V_{6} \cdot A a C}{K_{6}+A a C} \quad \text { actoacetyl-CoA } \rightarrow \text { butyryl-CoA } \\
& R_{7}^{\mathrm{A}}=\frac{V_{7} \cdot B C}{K_{7}+B C} \quad R_{7}^{\mathrm{S}}=\frac{V_{7}}{f_{7}} \frac{B C}{K_{7}+B C} \quad \text { butyryl-CoA } \rightarrow \text { butyrate } \\
& R_{8}^{\mathrm{A}}=0 \quad R_{8}^{\mathrm{S}}=\alpha_{81} \cdot B C \cdot A d h E 1 \quad \text { butyryl-CoA } \rightarrow \text { butanol } \\
& R_{9}^{\mathrm{A}}=\alpha_{9} \cdot A \cdot A a C \cdot C t f^{\mathrm{A}} \quad R_{9}^{\mathrm{S}}=\alpha_{9} \cdot A \cdot A a C \cdot C t f^{\mathrm{s}} \quad \text { acetate } \rightarrow \text { acetyl-CoA } \\
& \text { inactive in Cac-ctfA398s::CT } \\
& R_{10}^{\mathrm{A}}=\alpha_{10} \cdot B \cdot A a C \cdot C t f^{\mathrm{A}} \quad R_{10}^{\mathrm{s}}=\alpha_{10} \cdot B \cdot A a C \cdot C t f^{\mathrm{s}} \quad \begin{array}{l}
\text { butyrate } \rightarrow \text { butyryl-CoA } \\
\text { inactive in Cac-ctfA398s::CT }
\end{array} \\
& R_{11}^{\mathrm{A}}=\alpha_{11} \cdot A d c^{\mathrm{A}} \cdot A a C \quad R_{11}^{\mathrm{S}}=f_{11} \cdot \alpha_{11} \cdot A d c^{\mathrm{s}} \cdot A a C \quad \text { acetoacetyl-CoA } \rightarrow \text { acetoacetate }
\end{aligned}
$$

where Michaelis-Menten-like expressions, e.g., $R_{4}$ were employed if no significant changes in enzyme concentrations between both metabolic states were reported (Janssen et al. 2010) and a multiplication of kinetic parameter, intermediate, and enzyme concentration otherwise, e.g., $R_{5}$. The model parameters are given in Appendix 4, Table 2. The superscripts 'A' and ' $S$ ' denote acidogenic and solventogenic enzyme concentrations that are calculated as steady states from estimated acidogenic $\left(r_{E}^{\mathrm{A}}\right)$ and solventogenic $\left(r_{E}^{\mathrm{s}}\right)$ production rates using the equation

$$
\frac{d E_{i}}{d t}=r_{E_{i}}^{\{\mathrm{A}, \mathrm{S}\}}-D \cdot E_{i} .
$$

There is little information available on the changes in the proteome during the metabolic shift in phosphate-limited continuous cultures (Schaffer et al. 2002). Accordingly, because their deduction from transcriptional data might be misleading (Keene 2010; Straub 2011; Zhang et al. 2014), here we assign constant, but phenotype-specific, intracellular enzyme concentrations to the subpopulations. In particular, significantly higher amounts of solvent-forming enzymes are present in solventogenic cells which is in accordance with experimental observations from acidogenic and solventogenic steady states (Fontaine et al. 2002; Janssen et al. 2010).

$\mathrm{pH}$-dependent specific enzymatic activities strongly regulate product formation (Andersch et al. 1983; Dürre et al. 1995; Jones and Woods 1986). Interestingly, experimental investigation of catalytic efficiencies has revealed that several enzymes operate optimally for either acidogenic or solventogenic intracellular $\mathrm{pH}$ levels and exhibit significant changes in their specific activities between both metabolic phases (Andersch et al. 1983; Hartmanis et al. 1984; Ho et al. 2009).

For the mutant Cac-ctfA398s::CT we assume that no active CoA-transferase is present in the cells and, thus, the concentrations $C t f^{\mathrm{A}}$ and $C t f^{\mathrm{s}}$ are zero. Consequently, the reactions $R_{9}, R_{10}$, and $R_{11}$, representing the unidirectional re-assimilation of acetate and butyrate, and the formation of acetoacetate , are impeded in Cac-ctfA398s::CT cells.

The induction patterns of aldehyde/alcohol dehydrogenases AdhE1/2 are remarkably different from that of other enzymes considered in the model. These enzymes, required for ethanol and butanol production, exhibit antagonistic expression (Grimmler et al. 2011; Janssen et al. 2010). The gene product of $a d h E 2$ is induced during acidogenesis (Grimmler et al. 2011) and is believed to facilitate only the formation of ethanol. In contrast, AdhE1 catalyses the production of both ethanol and butanol (Dürre et al. 1995; Walter et al. 1992) and is induced during solventogenesis (Fontaine et al. 2002; Grimmler et al. 2011). On the basis of these experimental findings, the model considers that acidogenic and solventogenic cells differ in their proteomic composition, including both AdhE1 and AdhE2, see Eqs. (4c) and (4h). However, whereas recent batch and continuous culture experiments have verified the crucial role of AdhE1 in the transition from acid- to solvent formation using the mutant $C$. acetobutylicum Cac-adhE1468s::CT, the mutant $C$. acetobutylicum Cac-adhE2696s::CT continues to form ethanol and, thus, challenges our current view on clostridial 
ethanol production (Cooksley et al. 2012). Acidogenic- and solventogenic-specific kinetic parameters were introduced for those enzymes exhibiting significant changes of their specific activities. Experimental evidence notably indicates that the formation of acetate (Andersch et al. 1983; Hartmanis et al. 1984), Eq. (4b), acetone (Ho et al. 2009), Eq. (4e), and butyrate (Andersch et al. 1983; Wiesenborn et al. 1989b), Eq. (4g) are subject to pH-dependent kinetic regulation, which is reflected in the additional factors $f_{2}, f_{7}$, and $f_{11}$ to take into account the relative changes of the kinetic parameters.

In agreement with experimental evidence, we assume that the glycolytic backbone of $\mathrm{ABE}$ fermentation is independent of the $\mathrm{pH}$ level resulting in reaction rates independent from cellular phenotype, see Eqs. (4a), (4d), and (4f).

A further expectation is that the ctfA mutant adjusts the metabolic flow to accommodate its cellular demands, i.e., inactivation of acetone formation is compensated for by adapting the flow to other metabolic products. In the solvent-forming wild type (Haus et al. 2011; Millat et al. 2013b), acetone formation is responsible for approximately $22 \%$ of all measured fermentation products, whereas it represents only 3\% during acidogenesis (Millat et al. 2013a). Thus, changes in response to the blockade of acetone formation should be more apparent under solventogenic conditions. Lütke-Eversloh and Bahl (2011) have speculated that the intracellular concentrations of ATP and $\mathrm{NADH}$ (and their energetically reduced forms) play a crucial role in these regulatory changes. Because the underlying mechanisms and principles are yet unknown, the regulatory effects were mimiced by changing the parameters, estimated for the wild type (Millat et al. 2013b), such that the steady-state concentrations of the forward-shift experiments using Cac-ctfA398s::CT cells are reproduced. For the sake of comparison, the simulation using wild-type parameters is shown in the Appendix 3.

To elucidate the existence of an alternative re-assimilation mechanism for butyrate, we compare two models: First, a ctfA mutant without alternative pathways for butyrate re-assimilation and, second, a ctfA mutant in which an active $\mathrm{pH}$-dependent reverse butyrate branch is operational. For the latter model, we add a $\mathrm{pH}$-dependent reverse reaction to Eq. (4j). Here, for the sake of simplicity, a potential $\mathrm{pH}$-dependence of the forward reaction from butyryl-CoA to butyrate was neglected. Then, the rate equation

$$
R_{7}=\frac{V_{7} \cdot B C}{K_{7}+B C}-\frac{V_{7}^{\mathrm{r}} \cdot B}{K_{7}+B} f(p H)
$$

is obtained for the formation and re-assimilation of butanol. The $\mathrm{pH}$-dependent factor

$$
f(p H)=-(p H-5.7) / 1.2
$$

activates the reverse reaction in response to the changing $\mathrm{pH}$ level. Due to the lack of detailed information about the $\mathrm{pH}$-dependent activity of the reactions, $f$ is restricted to $[0,1]$ and follows the time course of the $\mathrm{pH}$ level directly. Note that Eq. (5) describes the acidogenic and the solventogenic phenotype which differ in the activity of butyrate re-assimilation. As a consequence, the above equation describes the butyrate re-assimilation during the $\mathrm{pH}$-shift and a reduced butyrate formation during solventogenesis. Both scenarios are compared to experimental data in the following section.

\section{Comparison of simulation and experimental data}

The presented continuous culture experiments (Fig. 2) had revealed that the CoA-transferase mutant Cac-ctfA398s::CT is able to shift its metabolism from acid-production to solvent-production, see also (Cooksley et al. 2012; Lehmann et al. 2012a) for similar results using batch cultures. Importantly and in accordance with our expectations, this mutant is unable to form acetone during both phases.

To start with, we briefly summarise the changes in the product formation attributed to, yet unknown, regulatory mechanisms. Towards this end, we applied the wild-type model (Millat et al. 2013b) taking into account the mutation of the CoA-transferase and the changed $\mathrm{pH}$-dependent 
growth behaviour, but no changes to the kinetic parameters were introduced. A comparison between the numerical simulation of this adjusted wild-type model and experimental data for the $c t f A$ mutant is shown in Fig. 4 in Appendix 3.

During acidogenesis, the predicted acetate concentration coincides with the measured data. Additionally, the decline of acetate after the $\mathrm{pH}$-shift is reproduced by the wild-type model. However, major deviations occur during solventogenesis, where the experimentally observed acetate formation is tripled in comparison to prediction. While the predicted butyrate formation under acidogenic conditions is only slightly higher $(\sim 1.2$-fold $)$ than in the experiments, the adjusted wild-type model predicts a much higher ( 6-fold) butyrate formation under solventogenic conditions. This might result from the high affinity of CtfA/B towards butyrate obtained from parameter estimation in (Millat et al. 2013b), which is inconsistent with biochemical studies (Wiesenborn et al. 1989b). This activitiy is balanced in the wild-type model by an increased butyrate formation activity resulting in an overestimation of butyrate levels in the ctfA mutant.

The qualitative behaviour of the measured ethanol concentration is well reproduced by the adjusted wild-type model, but, interestingly, it is almost doubled in comparison to the simulation. This increase could represent a further adaptation to the mutation in response to pertubations downstream of the acetyl-CoA branch point, as reported for a 3-hydroxybutyryl-CoA-dehydrogenase-negative mutant (Lehmann and Lütke-Eversloh 2011).

Importantly, the observed butanol production is only reduced to approximately two-thirds of the value predicted by the adjusted wild-type model. This indicates that during continuous cultivation (and in contrast to batch cultivation (Cooksley et al. 2012; Lehmann et al. 2012a)) butanol formation is not greatly inhibited by disruption of the acetone formation as proposed by (Hartmanis et al. 1984). Furthermore, the contribution of butanol to the pool of fermentation products seems to be less affected by the mutation.

An analysis of the molar amounts of liquid products measured in the experiments showed that butanol represented about $34 \%$ (39.5\% in the wild type) of the final metabolites during solventogenesis. The proportions of butyrate and ethanol, however, are almost doubled in comparison to the wild type. Strikingly, acetate produced by the mutant represents $45 \%$ of liquid products. This almost coincides with sum of $47.3 \%$ of acetone $(32.8 \%)$ and acetate (14.5\%) observed in the wild type. All reference data are taken from simulations in (Millat et al. 2013b).

Taking into account the adapted formation rates of the fermentation products in the ctfA mutant, we simulated two ctfA-mutant models: 1. CoA-transferase-dependent re-assimilation, relying exclusively on $\mathrm{Ctf} A / \mathrm{B}$ for acetate and butyrate re-assimilation (and thus reflecting the classical view of $\mathrm{ABE}$ fermentation (Dürre et al. 1995; Hartmanis and Gatenbeck 1984), and; 2. CoA-transferase independent re-assimilation, taking into account an alternative butyrate re-assimilation suggested in (Desai et al. 1999; Lehmann et al. 2012a). These two models differ in the representation of butyrate formation, which is either described by Eq. (4j), that assumes a completely disabled acid re-assimilation, or Eq. (5), that considers a reverse mechanism from butyrate to butyryl-CoA. In accordance with the introduced ctfA mutation, the CoA-transferase-mediated reactions $R_{9}$ and $R_{10}$, which represent the CtfA/B-dependent re-assimilation, are inactive in both models.

Comparing the experimental data with the predicted time courses for the mutant, we found that the two $c t f A$-mutant models equally well reproduce the experimentally observed behaviour of the acetate concentration and, in particular, its decline during the $\mathrm{pH}$-induced metabolic switch (Fig. 2[a]). In fact, both ctfA-mutant models coincide for the chosen set of parameters. Consequently, the $\mathrm{pH}$-dependent population growth and the continuous wash-out are responsible for the drop of the concentration after the initiation of the $\mathrm{pH}$-shift. This led us conclude that the mutant Cac-ctfA398s::CT is unable to re-assimilate acetate under the conditions we used. Both models reproduce the experimentally observed loss of acetone formation in the mutant. Furthermore, the predicted ethanol formation agrees with the experimental data. 

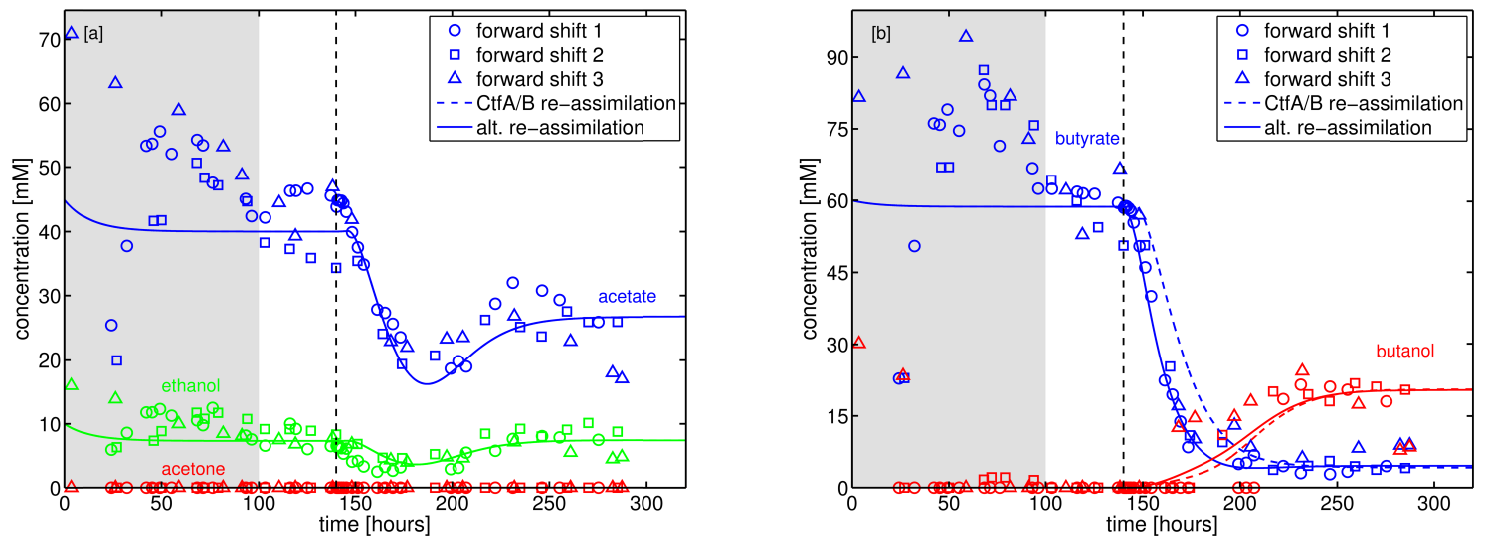

Fig. 2 Comparison of experimental data from three independent forward-shift experiments (symbols) and simulations for the mutant strain Cac-ctfA398s::CT: [a] acetate (blue), ethanol (green), and acetone (red); [b] butyrate (blue) and butanol (red). The simulations of a model with a CtfA/B-dependent re-assimilation of acetate and butyrate (CtfA/B re-assimilation, dashed lines) and the model assuming an alternative, but CtfA/B-independent, butyrate re-assimilation mechanism (alt. re-assimilation, solid lines) are plotted. The vertical dashed line indicates the initiation of the $\mathrm{pH}$-shift at $t=140 \mathrm{~h}$ and the grey-shaded area an initial growth phase that is excluded from our model.

In Fig. 2[b], the simulations of the two mutant models are compared with experimental data with respect to butyrate and butanol production. These data show that the butyrate concentration declined earlier and faster than predicted by the model only assuming a CtfA/B-dependent re-assimilation of butyrate. As a consequence, a reversible CtfA/B-independent mechanism was assumed that reduces the butyrate concentration during the $\mathrm{pH}$-induced shift in addition to the $\mathrm{pH}$-dependent population growth and continuous wash-out. Furthermore, the experimentally observed data suggest that the activity of this reaction is $\mathrm{pH}$-dependent becoming active before the acidogenic population drops.

When this alternative mechanism is incorporated into our model, the experimentally observed decline in butyrate concentration is very well reproduced. This suggests that an alternative CtfA/B-independent butyrate re-assimilation pathway does indeed exist in C. acetobutylicum. In the present manuscript, we assumed a reverse reaction of the butyrate kinase, Buk, and the phosphotransbutyrylase, Ptb, as proposed by Hüsemann and Papoutsakis (1989). At solventogenic conditions, the reverse reaction reduces the net formation of butyrate by 7 -fold due to its competitive effect on butyrate formation, see Eq. (5). This agrees with the result reported in (Andersch et al. 1983), where a 6-fold change of the specific butyrate kinase activity was observed.

The CtfA/B-independent model further predicts a slightly faster rise of the butanol concentration due to the increased intracellular concentration of butyryl-CoA than the CtfA/B-dependent model which lacks re-assimilation activity. However, the experimentally observed butanol formation increased faster and earlier than predicted by the simulations. In particular, the increase of the butanol concentrations starts before the solventogenic population significantly contributes to its formation. A potential explanation for that observation could be the sequential action of an aldehyde:ferredoxin oxidoreductase and a butanol dehydrogenase as alternative route of butyrate re-assimilation and subsequent butanol formation as discussed in more detail in the following section.

\section{Discussion}

The Gram-positive bacterium C. acetobutylicum switches its metabolism according to environmental $\mathrm{pH}$ levels between two distinct phases that are characterised by their main metabolic products. During acidogenesis $(\mathrm{pH}>5.2)$, the formation of acids (acetate and butyrate) dominates, whereas the $\mathrm{pH}$-neutral solvents acetone and butanol are predominantly formed during solventogenesis $(\mathrm{pH}<5.1)$ in continuous culture under phosphate limitation. Ethanol is produced in only minor amounts. It is believed that this phenomenon represents a clostridial regulatory process to prevent further acidification of the environment, which eventually results in a life threatening 
situation, and is part of the clostridial pH-induced stress response (Dürre 2007; Jones and Woods 1986). Furthermore, the excreted acids are known to be toxic, so that solventogenesis could be also induced for detoxification of the surrounding medium (Rogers and Gottschalk 1993). Batch experiments indicate that the initiation of the metabolic switch from acidogenesis to solventogenesis in C. acetobutylicum involves several measures: the specific activity of acid-forming enzymes is reduced and that of solvent-forming enzymes is increased, formerly excreted acids are re-assimilated, and the metabolic flow through the branch points is re-routed to $\mathrm{pH}$-neutral solvents (Dürre 2005). Biochemical investigations (Hartmanis and Gatenbeck 1984); Wiesenborn et al. 1989a) have suggested that the hetero-dimeric CoA-transferase, CtfA/B, mediates the re-assimilation of the acids acetate and butyrate and the formation of acetoacetate from acetoacetyl-CoA. However, several investigations have led to the notion that a CtfA/B-independent butyrate re-assimilation mechanism is also present in C. acetobutylicum (Desai et al. 1999; Lehmann et al. 2012a; Lehmann et al. 2012b). It has been suggested by Hüsemann and Papoutsakis (1989), that a reverse pH-dependent activity of the butyrate kinase, Buk, and the phosphotransbutyrylase, Ptb, which facilitate the butanol formation in the forward direction, might provide this alternative mechanism. Assuming that the $\mathrm{pH}$-optimum of the reverse reaction lies at solventogenic $\mathrm{pH}$ levels, it would also result in a reduction of the net butyrate formation rate at solventogenic conditions.

In the present study, we applied a systems biology approach combining systematic experiments in continuous culture, data processing, and modelling to test the hypothesis of a $\mathrm{CtfA} / \mathrm{B}$-independent re-assimilation of butyrate. Towards this end, three independent forward-shift experiments using the mutant Cac-ctfA398s::CT in a chemostat under phosphate limitation were conducted. Over the entire time course of the experiment, the $\mathrm{pH}$ level, the optical density, and the fermentation products were measured. Afterwards, the measured data were used to model the $\mathrm{pH}$-induced metabolic switch in the mutant strain based on a model established for the wild type using the same standard operational procedures (Millat et al. 2013b). Importantly, our model separates population dynamics and metabolic dynamics. Thus, it is capable of distinguishing between changes in the population size and changes in the network of ABE fermentation, of which might induce the observed decline of the acids during the $\mathrm{pH}$-induced metabolic shift in continuous cultures. Furthermore, our standardised experimental setup separates the $\mathrm{pH}$-shift and the resultant cellular adaptation in time. This separation of timescales allows our modelling approach to distuinguish between a reverse mechanism activated by changing $\mathrm{pH}$-levels (which are immediate) and transcriptional changes that the solventogenic subpopulation is responsible for.

From the experiments and the theoretical investigations we derive four major conclusions: Firstly, active CoA-transferase is mandatory for acetone formation and there is no mechanism present in $C$. acetobutylicum to circumvent the conversion of acetoacetyl-CoA to acetoacetate. This finding confirms recent observations in batch cultures (Cooksley et al. 2012; Lehmann et al. 2012a) and is additionally supported by recent experiments showing that CtfA/B, not Adc, is the rate-determining enzyme for acetone formation (Tummala et al. 2003a; Tummala et al. 2003b). Note that $a d c$ mutants still form minor amounts of acetone (Cooksley et al. 2012; Lehmann et al. 2012a) due to a non-enzymatic spontaneous decarboxylation of acetoacetate (Han et al. 2011).

Secondly, an alternative re-assimilation mechanism for butyrate, but not for acetate, is present and active in C. acetobutylicum. As a consequence, the decline of acetate in the mutant after the initiation of the $\mathrm{pH}$-shift is explained by the shift in the prevalent subpopulation. In contrast, the observed decline of butyrate requires an additional reducing process in the model that is interpreted as an CtfA/B-independent mechanism (Desai et al. 1999; Lehmann et al. 2012a; Lehmann et al. 2012b). In the present manuscript, we have accommodated previous suggestions (Hüsemann and Papoutsakis 1989) and assumed a reverse activity of butyrate kinase and phosphotransbutyrylase. However, we cannot exclude that another, as yet uncharacterised mechanism is responsible for the observations reported here. The existence of such a mechanism is also supported by batch culture experiments using a $p t b$ mutant which observed butyrate re-assimilation even without Ptb activity (Lehmann 2012b). The concerted action of a putative aldehyde:ferredoxin oxidoreductase (AOR, 
CAC2018) and NADH-dependent butanol dehydrogenases, BdhA and BdhB (Petersen et al. 1991; Welch et al. 1989), could even bypass the indermediate butyryl-CoA and result in an immediate increase of butanol as a direct stress response to the rapidly changing $\mathrm{pH}$ level. Whereas $b d h A$ and $b d h B$ are arranged in monocistronic operons in the chromosome, in each case controlled by a single promoter (Walter et al. 1992), computational predictions using the published annotated genome (Nölling et al. 2001) suggest that AOR forms an operon in the clostridial chromosome that includes several enzymes for fatty acids biosynthesis, two enoyl-CoA hydratases, and a 3-hydroxyacyl-CoA dehydrogenase (Caspi et al. 2012; Dale et al. 2010; Karp et al. 2010). Importantly, the butanol dehydrogenases possess much higher activity with butyraldehyde than with acetaldehyde, dropping sharply from their maximum at $\mathrm{pH}=5.5$ to less than $50 \%$ with changes of $\pm 0.7 \mathrm{pH}$ unit (Petersen et al. 1991). Hence, this pathway is only able to prevent intracellular accumulation of butyryl-CoA over a limited range of the $\mathrm{pH}$. Under more solventogenic $\mathrm{pH}$ levels, C. acetobutylicum has to establish other means to adapt to the new environmental conditions, including the switch of its metabolism to the formation of the $\mathrm{pH}$ neutral solvents acetone and butanol. These considerations are the subject of ongoing investigations. This suggests that the alternative butyrate re-assimilation mechanism potentially includes butanol formation in acidogenic cells, which is currently not considered in our models, to prevent intracellular accumulation of butyryl-CoA.

Thirdly, the CtfA/B-independent uptake mechanism is activated by the drop of the $\mathrm{pH}$ level and re-assimilates butyrate before the acidogenic population declines. Accordingly, we have assumed that the reverse activity is directly coupled to the external $\mathrm{pH}$. Furthermore, the solventogenic population increase is delayed with respect to the $\mathrm{pH}$-shift. Thus, it contributes less to the product formation during the first 20 hours after the initiation of the $\mathrm{pH}$-shift.

Finally, the proposed multifunctional role of the CoA-transferase during the $\mathrm{pH}$-induced phase transition in clostridial ABE fermentation has to be challenged. Commonly, it is believed that this enzyme is crucial for the re-assimilation of the acids acetate and butyrate, their conversion to their respective CoA-derivates, and the formation of acetoacetate, the precursor of acetone (Dürre et al. 1995). However, the results presented here suggest that the butyrate re-assimilation during the metabolic shift in phosphate-limited continuous cultures could be explained by an alternative $\mathrm{CtfA} / \mathrm{B}$-independent mechanism. Furthermore, no significant acetate re-assimilation occurs in the wild type during the metabolic shift under our experimental conditions (Fig. 7 in Millat et al. (2013b)). Consequently, a mathematical model assuming an alternative re-assimilation of butyrate and a $\mathrm{pH}$-dependent reduced acetate formation rate, but no CtfA/B-mediated acid re-assimilation, could explain the observations reported in Haus et al. (2011) and Millat et al. (2013b).

Bearing in mind that phosphate-limited continuous cultures of the ATCC 824 wild type generate acetone at low concentrations under acidogenic conditions, it follows that small amounts of the CoA-transferase have to be present in the culture during that metabolic phase. In turn, some expression of the sol operon should be expected. According to our definition, such an expression profile characterises the solventogenic phenotype. Our findings support the previously suggested hypothesis (Clarke et al. 1988; Millat et al. 2013b) that, at least in continuous culture, a small solventogenic subpopulation is present under acidogenic conditions. Furthermore, a recently published elementary mode analysis reported that 'stressed' batch cultures of C. acetobutylicum simultaneuously exhibit acidogenic and solventogenic characteristics (Kumar et al. 2014), which could result from the superposition of those coexisting acid- and solvent-forming subpopulations.

Using the CoA-transferase mutant Cac-ctfA398s::CT, we demonstrated here how a systems biology approach improves our knowledge of the ABE fermentation in C. acetobutylicum. Recently reported improvements and new developments in genetic tools for the manipulation of Clostridia (Al-Hinai et al. 2012; Kuehne and Minton 2012; Kuehne et al. 2011, Lütke-Eversloh 2014) offer new perspectives for the investigation of the ABE network. Using currently available mutants growing in batch cultures, several authors have observed a remarkable flexibility of C. acetobutylicum to overcome perturbations in its metabolic network (Cooksley et al. 2012; Lehmann and Lütke-Eversloh 2011; Lehmann et al. 2012a). Importantly, the assumption in our model that AdhE1 is essential for the clostridial transition to solventogenesis is supported by batch 
culture (Cooksley et al. 2012) and continuous culture (data not shown) experiments. These results as well as the data presented in this manuscript demonstrate that $C$. acetobutylicum is capable of compensating several mutations by adjusting the relative amounts of the (remaining) fermentation products. However, the underlying regulatory mechanisms are poorly understood. It is speculated that the intracellular ATP and NADH pools play a crucial role in the clostridial adaptation to changing environmental condition including the $\mathrm{pH}$ level (Girbal and Soucaille 1994; Wietzke and Bahl 2012), nutritional composition (Bahl et al. 1986; Girbal and Soucaille 1994), and further limitations (e.g. iron (Junelles et al. 1988; Vasileva et al. 2012)).

An essential prerequisite for quantitative analysis of experimental data using mathematical models is a standardised experimental setup. In particular, the experimental design has to allow for a legitimate comparison between different experiments. As we have demonstrated here, such an approach enables the systematic testing of hypotheses regarding the metabolic switch of C. acetobutylicum, such as investigating the dynamic re-assimilation of acids in metabolically impaired mutants. The majority of newly created mutants represent cells in which a particular enzyme activity involved in the ABE fermentation has been ablated. In the future, modelling of the $A B E$ pathway would significantly benefit from the generation of comparable strains in which these same enzymes are deliberated over-produced.

\section{Acknowledgements}

The authors acknowledge support by the German Federal Ministry for Education and Research (BMBF FKZ 0315782D) and by the Biotechnology and Biological Sciences Research Council (UK) (BBSRC No. BB/I004475/1), as part of the European Transnational Network 'Systems Biology of Microorganisms' (SysMo) within the COSMIC consortium. N.P.M. also acknowledges funding received from the Biotechnology and Biological Sciences Research Council, as part of the BBSRC Sustainable Bioenergy Centre (BSBEC) initiative, for the programme 'Second Generation, Sustainable Biofuels' (BBSRC No. BB/G016224/1). We thank Ulf W. Liebal and Carola Berger for their critical comments and fruitful discussions. The responsibility for the content of this manuscript lies with the authors.

\section{Conflict of interests}

The authors declare that they have no conflict of interest.

\section{Appendix 1}

Time course of external $\mathrm{pH}$ and joint experimental data for forward-shift experiments in a chemostat using the mutant Cac-ctfA398s::CT

The external $\mathrm{pH}$ level was the only environmental parameter varied during the forward-shift experiments considered in the present manuscript. The clostridial culture grew initially at an external $\mathrm{pH}$ level of 5.7. After the acidogenic culture approached a steady state, the $\mathrm{pH}$ was shifted to 4.5. The time course of the $\mathrm{pH}$ was recorded during the experiments.

Assuming that the initiation of the $\mathrm{pH}$-drop is much faster than any induced adaptation, we model the decline in the $\mathrm{pH}$ level using an exponential function which is determined by the two parameters $\alpha$ and $\beta$. Here, $\alpha$ describes the time constant of the $\mathrm{pH}$-drop and $\beta$ the initiation of the $\mathrm{pH}$-shift. Then, the time course of the external $\mathrm{pH}$ is represented as

$$
p H(t)= \begin{cases}5.7 & \text { for } t<\beta \\ 1.2 \cdot \mathrm{e}^{-\alpha(t-\beta)}+4.5 & \text { for } t \geq \beta\end{cases}
$$

According to the chosen experimental setup, the $\mathrm{pH}$ varies between acidogenic conditions at $\mathrm{pH}=5.7$ and solventogenic conditions at $\mathrm{pH}=4.5$. The parameters estimated from the dynamic shift experiments are given in Table 1. They are used to join the three experiments into a single representation by shifting and scaling operations as reported in (Millat et al. 2013b). Finally, we 
apply the parameters $\bar{\alpha}=0.135 \mathrm{~h}^{-1}$ and $\bar{\beta}=140 \mathrm{~h}$ to re-scale the joint data into the common observation time scale. Subsequently, this data set is used for parameter estimation and comparison of experimental data and simulation.

Table 1 Estimated parameters of Equation (6) for the dynamic shift experiments using the mutant Cac-ctfA398s::CT

\begin{tabular}{lll}
\hline Experiment & $\alpha\left[\mathrm{h}^{-1}\right]$ & $\beta[\mathrm{h}]$ \\
\hline First & 0.138 & 115.8 \\
Second & 0.138 & 113.0 \\
Third & 0.185 & 120.5 \\
\hline
\end{tabular}

\section{Appendix 2}

Estimation of $\mathrm{pH}$-dependent population growth

Following the assumption that the clostridial population consists of an acidogenic and a solventogenic subpopulation (Millat et al. 2013b), we represent the optical density as the sum

$$
O D(t)=O D^{\mathrm{A}}(t)+O D^{\mathrm{S}}(t)
$$

of the time-dependent densities of both subpopulations.

The solventogenic culture grows exponentially after the initiation of the $\mathrm{pH}$-shift and approaches the solventogenic steady state $\mathrm{OD}^{\mathrm{S}}=2.4$ as found in experiments. Such behaviour is represented by a hyperbolic tangent

$$
O D^{\mathrm{S}}(t)=\frac{\mathrm{OD}^{\mathrm{S}}}{2}\left(\tanh \left[\alpha_{\mathrm{s}}\left(t-\beta_{\mathrm{s}}\right)\right]+1\right)
$$

which is an alternative representation for logistic growth. The parameter $\alpha^{\mathrm{s}}=0.087 \mathrm{~h}^{-1}$ measures the maximal growth rate of the solventogenic population, whereas $\beta^{\mathrm{s}}=190 \mathrm{~h}$ determines the time of maximal growth. Note that the mutant grows slower than the wild type which is characterised by the parameters $\alpha^{\mathrm{S}}=0.175 \mathrm{~h}^{-1}, \beta=178 \mathrm{~h}$, and $\mathrm{OD}^{\mathrm{S}}=5.0$ (Millat et al. 2013b). The ratio of the growth rates of the mutant and the wild type coincides with the respective ratio of the optical densities at solventogenic steady state.

In contrast to the solventogenic subpopulation, the decline of the acidogenic subpopulation follows an exponential curve

$$
O D^{\mathrm{A}}(t)=\mathrm{OD}^{\mathrm{A}} \cdot \exp \left\{-\alpha_{\mathrm{A}}\left(t-\beta_{\mathrm{A}}\right)\right\}
$$

that starts at the acidogenic optical density $\mathrm{OD}^{\mathrm{A}}=4.2$. Its time constant $\alpha^{\mathrm{A}}=0.072 \mathrm{~h}^{-1}$ almost fits the dilution rate $D=0.075 \mathrm{~h}^{-1}$ within the experimental uncertainty. The acidogenic population starts to drop at $\beta^{\mathrm{A}}=146 \mathrm{~h}$ and, thus, with a delay of 6 hours after the initiation of the $\mathrm{pH}$-shift at $t=140 \mathrm{~h}$. These findings are in agreement with the results reported in (Millat et al. 2013b). The reduced optical density suggests that the mutant Cac-ctfA398s::CT grows slower at acidogenic conditions in comparison to the wild type $\left(\mathrm{OD}^{\mathrm{A}}=5.5\right.$ (Millat et al. 2013b)). In Fig. 3, the experimental data of three independent forward-shift experiments, joint according to the method described above, are compared to the fitting function (7).

In the present manuscript, we assumed that the clostridial culture is homogeneous at acidogenic and solventogenic steady states and heterogeneous during the $\mathrm{pH}$-induced metabolic shift. 


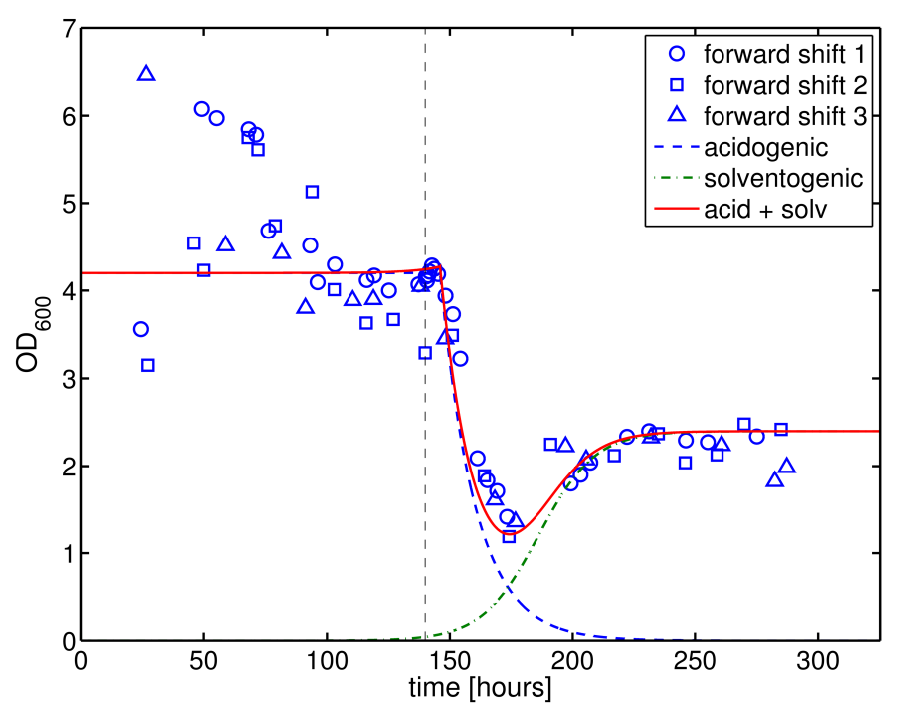

Fig. 3 Observed and fitted optical density $\left(\mathrm{OD}_{600}\right)$ of Cac-ctfA398s::CT populations grown in continuous culture under phosphate limitation over the observation time. The blue dashed line represents the acidogenic population which exponentially declines with a delay of 6 hours after the initiation of the $\mathrm{pH}$-shift. The solventogenic population rises in response to the changing $\mathrm{pH}$ following a logistic growth curve, green dashed-dotted line. The sum of the two populations is shown as solid red line. The $\mathrm{pH}$-shift was initiated at $t=140 \mathrm{~h}$ as denoted by the vertical dashed line.

\section{Appendix 3}

Application of the adjusted wild-type model for the mutant Cac-ctfA398s::CT

To specify the changes induced by the knock-out of the ctfA gene, we first applied our two-population model developed for the wild-type strain (Millat et al. 2013b) without consideration of intracellular adaptions. Towards this end, we only integrated the $\mathrm{pH}$-dependent population growth observed for the mutant (Appendix 2) and an inactive CoA-transferase reaction, but used unchanged wild-type parameters for all other biochemical reactions. The corresponding numerical simulation is shown in Fig. 4. Whereas the experimentally observed acidogenic acetate formation and its drop during the $\mathrm{pH}$-induced shift are well reproduced, the acetate concentration during solventogenesis is significantly underestimated by the simulation. The observed butyrate concentration during acidogenic conditions is slightly lower than predicted, but importantly the adjusted wild-type model fails to fit the $\mathrm{pH}$-induced drop of butyrate and its solventogenic formation. The formation of acetone is completely abolished as a consequence of mutation. Furthermore, significant differences between simulated and observed product concentrations under solventogenic conditions are obvious. Whereas acetate concentrations are underestimated, the model overestimates butyrate formation. The measured ethanol production is doubled at both phases in comparison to the prediction of the wild-type model. Only minor changes occur under acidogenic conditions. We interpret these changes in product formation to be caused by a cellular response to compensate for the blocked acetone pathway. 

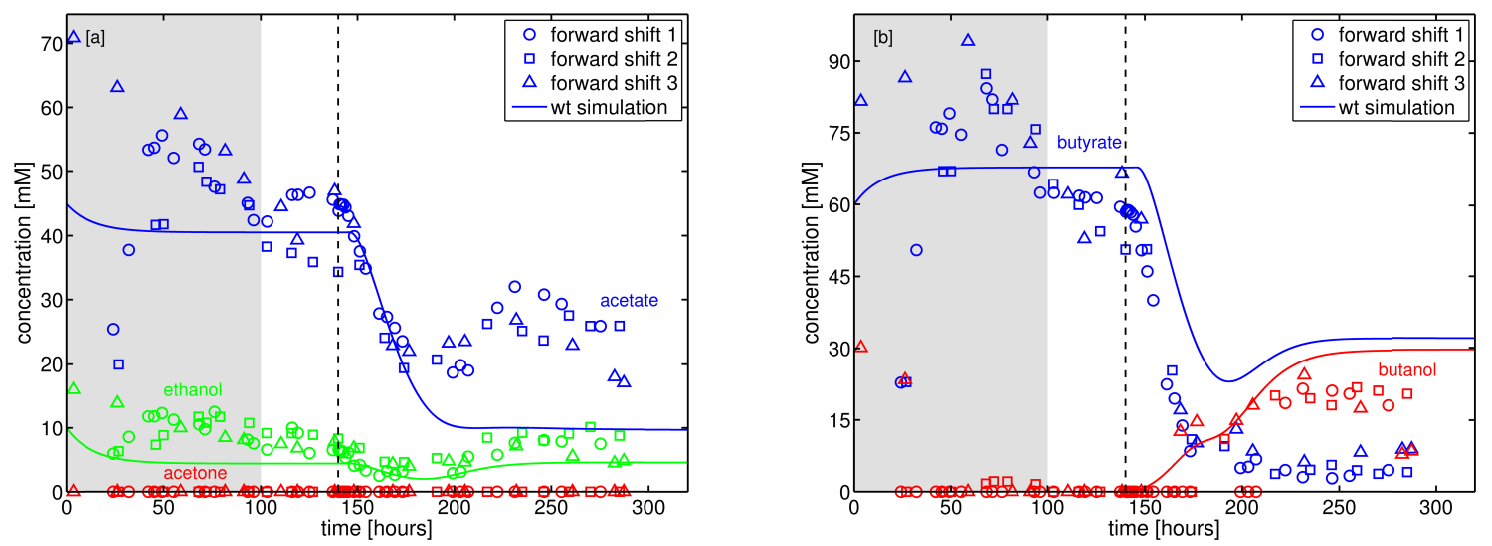

Fig. 4 Comparison of experimental data and the wild-type model. Here, all model parameters are chosen according to their original values estimated for the wild type (Millat et al. 2013b). The CoA-transferase is the only exception, whose activity is set to zero. The vertical dashed line indicates the initiation of the $\mathrm{pH}$-shift at $t=140 \mathrm{~h}$ and the grey-shaded area represents an initial growth phase that is excluded from our model.

\section{Appendix 4}

Model parameters

Table 2 Kinetic parameters. Limiting rates $V_{i}$ are in $\mathrm{mMh}^{-1}$, Michaelis-Menten constants $K_{i}$ in $\mathrm{mM}$, and parameters $\alpha_{i}$ in $\mathrm{mM}^{-1} \mathrm{~h}^{-1}$ and $\mathrm{mM}^{-2} \mathrm{~h}^{-1}$, respectively. The dimensionless parameters $f_{i}$ describe $\mathrm{pH}$-dependent changes of the specific activity.

\begin{tabular}{ccc}
\hline Parameter & Value & Reaction \\
\hline$V_{1}$ & 9.17 & glucose $\rightarrow$ acetyl-CoA \\
$K_{1}$ & 0.317 & \\
$V_{2}$ & 85 & acetyl-CoA $\rightarrow$ acetate \\
$K_{2}$ & 2.45 & \\
$f_{2}$ & $\mathbf{0 . 8}$ & \\
$\alpha_{31}$ & $\mathbf{0 . 1}$ & acetyl-CoA $\rightarrow$ ethanol (AdhE1) \\
$\alpha_{32}$ & $\mathbf{0 . 2 0 6}$ & acetyl-CoA $\rightarrow$ ethanol (AdhE2) \\
$V_{4}$ & 376.5 & acetyl-CoA $\rightarrow$ acetoacetyl-CoA \\
$K_{4}$ & 2.22 & \\
$\alpha_{5}$ & 0.0167 & acetoacetate $\rightarrow$ acetone \\
$V_{6}$ & 256.3 & acetoacetyl-CoA $\rightarrow$ butyryl-CoA \\
$K_{6}$ & 1.2 & \\
$V_{7}$ & $\mathbf{4 . 4}$ & butyryl-CoA $\rightarrow$ butyrate \\
$K_{7}$ & $8.17 \times 10^{-5}$ & \\
$f_{7}$ & $\mathbf{8}$ & \\
$V_{7}^{\mathrm{r}}$ & $\mathbf{3 . 5}$ & \\
$\alpha_{8}$ & $\mathbf{0 . 0 1 7 5}$ & butyryl-CoA $\rightarrow$ butanol (AdhE1) \\
$\alpha_{9}$ & $\mathbf{0 . 0 1}$ & acetate $\rightarrow$ acetyl-CoA \\
$\alpha_{10}$ & 0.68 & butyrate $\rightarrow$ butyryl-CoA \\
$\alpha_{11}$ & 0.1 & acetoacetyl-CoA $\rightarrow$ acetoacetate \\
$f_{11}$ & 25 & \\
\hline & &
\end{tabular}




\begin{tabular}{ccc}
\hline$r_{A d c}^{\mathrm{A}}$ & 2.68 & Adc synthesis rate \\
$r_{A d c}^{\mathrm{s}}$ & 4.53 & \\
$r_{A d h E 1}^{\mathrm{A}}$ & 1.21 & AdhE1 synthesis rate \\
$r_{\text {AdhE1 }}^{\mathrm{s}}$ & 4.57 & \\
$r_{\text {AdhE2 }}^{\mathrm{A}}$ & 4.245 & AdhE2 synthesis rate \\
$r_{A d h E 2}^{\mathrm{s}}$ & 0.865 & \\
$r_{C f f A B}^{\mathrm{A}}$ & $\mathbf{0}$ & CtfA/B synthesis rate \\
$r_{C t f A B}^{\mathrm{s}}$ & $\mathbf{0}$ & \\
\hline
\end{tabular}

Parameters adapted in the mutant model are set in bold face and new parameters additionally set in italics.

\section{References}

Al-Hinai M, Fast A, Papoutsakis E (2012) A novel system for efficient isolation of double-crossover allelic exchange mutants in Clostridium enabling markerless chromosomal gene deletions and DNA integration. Appl Environ Microbiol 78:8112-8121. doi: 10.1128/AEM.02214-12

Andersch W, Bahl H, Gottschalk G (1983) Level of enzymes involved in acetate, butyrate, acetone and butanol formation by Clostridium acetobutylicum. Appl Microbiol Biotechnol 18:327-332. doi: 10.1007/BF00504740

Bahl H, Andersch W, Gottschalk G (1982) Continuous production of acetone and butanol by Clostridium acetobutylicum in a two-stage phosphate limited chemostat. Appl Microbiol Biotechnol 15:201-205. doi: 10.1007/BF00499955

Bahl H, Gottwald M, Kuhn A, Rale V, Andersch W, Gottschalk G (1986) Nutritional Factors Affecting the Ratio of Solvents Produced by Clostridium acetobutylicum. Appl Environ Microbiol 52:169-172

Caspi R, Altman T, Dreher K, Fulcher C, Subhraveti P, Keseler I, Kothari A, Krummenacker M, Latendresse M, Mueller L, Ong Q, Paley S, Pujar A, Shearer A, Travers M, Weerasinghe D, Zhang P, Karp P (2012) The MetaCyc database of metabolic pathways and enzymes and the BioCyc collection of pathway/genome databases. Nucleic Acids Res 40:D742-D753. doi: 10.1093/nar/gkr1014

Clarke K, Hansford G, Jones D (1988) Nature and significance of oscillatory behavior during solvent production by Clostridium acetobutylicum in continuous culture. Biotechnol Bioeng 32:538-544. doi: 10.1002/bit.260320417

Cooksley C, Zhang Y, Wang H, Redl S, Winzer K, Minton N (2012) Targeted mutagenesis of the Clostridium acetobutylicum acetone-butanol-ethanol fermentation pathway. Metab Eng 14:630-641. doi: 10.1016/j.ymben.2012.09.001

Dale J, Popescu L, Karp P (2010) Machine learning methods for metabolic pathway prediction. BMC Bioinf 11:15. doi: $10.1186 / 1471-2105-11-15$

Desai R, Harris L, Welker N, Papoutsakis E (1999) Metabolic Flux Analysis Elucidates the Importance of the Acid-Formation Pathways in Regulating Solvent Production by Clostridium acetobutylicum. Metab Eng 1:206-213. doi: 10.1006/mben.1999.0118

Dürre P (2005) Formation of Solvents in Clostridia. In: Dürre (ed) Handbook on Costridia, CRC Press, Boca Raton, pp 671-693

Dürre P (2007) Biobutanol: An attractive biofuel. Biotechnol J 2(12):1525-1534. doi: 10.1002/biot.200700168

Dürre P, Fischer RJ, Kuhn A, Lorenz K, Schreiber W, Stürzenhofecker B, Ullmann S, Winzer K, Sauer U (1995) Solventogenic enzymes of Clostridium acetobutylicum: catalytic properties, genetic organization, and transcriptional regulation. FEMS Microbiol Rev 17:251-262. doi: 10.1111/j.1574-6976.1995.tb00209.x

Fiedler T, Mix M, Meyer U, Mikkat S, Glocker M, Bahl H, Fischer RJ (2008) The Two-Component System PhoPR of Clostridium acetobutylicum Is Involved in Phosphate-Dependent Gene Regulation. J Bacteriol 190:6559-6567. doi: 10.1128/JB.00574-08

Fischer RJ, Oehmcke S, Meyer U, Mix M, Schwarz K, Fiedler T, Bahl H (2006) Transcription of the pst Operon of Clostridium acetobutylicum Is Dependent on Phosphate Concentration and pH. J Bacteriol 188:5469-5478. doi: 10.1128/JB.00491-06

Fontaine L, Meynial-Salles I, Girbal L, Yang X, Croux C, Soucaille P (2002) Molecular Characterization and Transcriptional Analysis of adhE2, the Gene Encoding the NADH-Dependent Aldehyde/Alcohol Dehydrogenase Responsible for Butanol Production in Alcohologenic Cultures of Clostridium acetobutylicum ATCC 824. J Bacteriol 184:821-830. doi: 10.1128/JB.184.3.821-830.2002

Girbal L, Soucaille P (1994) Regulation of Clostridium acetobutylicum metabolism as revealed by mixed-substrate steady-state continuous cultures: role of NADH/NAD ratio and ATP pool. J Bacteriol 176:6433-6438 
Grimmler C, Janssen H, Krauße D, Fischer RJ, Bahl H, Dürre P, Liebl W, Ehrenreich A (2011) Genome-Wide Gene Expression Analysis of the Switch between Acidogenesis and Solventogenesis in Continuous Cultures of Clostridium acetobutylicum. J Mol Microbiol Biotechnol 20:1-15. doi: 10.1159/000320973

Grupe H, Gottschalk G (1992) Physiological Events in Clostridium acetobutylicum during the Shift from Acidogenesis to Solventogenesis in Continuous Culture and Presentation of a Model for Shift Induction. Appl Environ Microbiol 58:3896-3902

Han B, Gopalan V, Ezeji T (2011) Acetone production in solventogenic Clostridium species: new insights from non-enzymatic decarboxylation of acetoacetate. Appl Microbiol Biotechnol 91:565-576. doi: 10.1007/s00253-011-3276-5

Hartmanis M, Gatenbeck S (1984) Intermediary Metabolism in Clostridium acetobutylicum: Levels of Enzymes Involved in the Formation of Acetate and Butyrate. Appl Environ Microbiol 47:1277-1283

Hartmanis M, Klason T, Gatenbeck S (1984) Uptake and activation of acetate and butyrate in Clostridium acetobutylicum. Appl Microbiol Biotechnol 20:66-71. doi: 10.1007/BF00254648

Haus S, Jabbari S, Millat T, Janssen H, Fischer RJ, Bahl H, King J, Wolkenhauer O (2011) A systems biology approach to investigate the effect of $\mathrm{pH}$-induced gene regulation on solvent production by Clostridium acetobutylicum in continuous culture. BMC Syst Biol 5:10. doi: 10.1186/1752-0509-5-10

Ho MC, Menetret JF, Tsuruta H, Allen K (2009) The origin of the electrostatic perturbation in acetoacetate decarboxylase. Nature 459:393-397. doi: 10.1038/nature07938

Hoskisson P, Hobbs G (2005) Continuous culture - making a comeback? Microbiol 151:3153-3159. doi: 10.1099/mic.0.27924-0

Hüsemann M, Papoutsakis E (1989) Comparison between in vivo and in vitro enzyme activities in continuous and batch fermentations of Clostridium acetobutylicum. Appl Microbiol Biotechnol 30:585-595. doi: 10.1007/BF00255364

Janssen H, Döring C, Ehrenreich A, Voigt B, Hecker M, Bahl H, Fischer RJ (2010) A Proteomic and Transcriptional View of Acidogenesis and Solventogenesis in Clostridium acetobutylicum in a Chemostat Culture. Appl Microbiol Biotechnol 87:2209-2226. doi: 10.1007/s00253-010-2741-x

Janssen H, Grimmler C, Ehrenreich A, Bahl H, Fischer RJ (2012) A transcriptional study of acidogenic chemostat cells of Clostridium acetobutylicum - Solvent stress caused by a transient n-butanol pulse. J Biotechnol 161:354-365. doi: 10.1016/j.jbiotec.2012.03.027

Jones D, Woods D (1986) Acetone-butanol fermentation revisited. Microbiol Rev 50:484-524.

Jones S, Paredes C, Tracy B, Cheng N, Sillers R, Senger R, Papoutsakis E (2008) The transcriptional program underlying the physiology of clostridial sporulation. Genome Biol 9:R114. doi: 10.1186/gb-2008-9-7-r114

Junelles A, Janati-Idrissi R, Petitdemange H, Gay R (1988) Iron effect on acetone-butanol fermentation. Curr Microbiol 17:299-303. doi: 10.1007/BF01571332

Karp P, Paley S, Krummenacker M, Latendresse M, Dale J, Lee T, Kaipa P, Gilham F, Spaulding A, Popescu L, Altman T, Paulsen I, Keseler I, Caspi R (2010) Pathway Tools version 13.0: integrated software for pathway/genome informatics and systems biology. Brief Bioinform 11:40-79. doi: 10.1093/bib/bbp043

Keene J (2010) The global dynamics of RNA stability orchestrates responses to cellular activation. BMC Biol 8:95. doi: $10.1186 / 1741-7007-8-95$

Kuehne S, Minton N (2012) ClosTron-mediated engineering of Clostridium. Bioengineered 3:247-254. doi: $10.1186 / 1741-7007-8-95$

Kuehne S, Heap J, Cooksley C, Cartman S, Minton N (2011) ClosTron-mediated engineering of Clostridium. Methods Mol Biol 765:389-407. doi: 10.4161/bioe.21004

Kumar M, Saini, S Gayen K (2014) Elementary mode analysis reveals that Clostridium acetobutylicum modulates its metabolic strategy under external stress. Mol BioSyst 10: 2090-2105. doi: 101039/c4mb00126e

Lehmann D, Lütke-Eversloh T (2011) Switching Clostridium acetobutylicum to an ethanol producer by disruption of the butyrate/butanol fermentative pathway. Metab Eng 13:464-473. doi: 10.1016/j.ymben.2011.04.006

Lehmann D, Hönicke D, Ehrenreich A, Schmidt M, Weuster-Botz D, Bahl H, Lütke-Eversloh T (2012a) Modifying the product pattern of Clostridium acetobutylicum. Appl Microbiol Biotechnol 93:1-12. doi: 10.1007/s00253-011-3852-8

Lehmann D, Radomski N, Lütke-Eversloh T (2012b) New insights into the butyric acid metabolism of Clostridium acetobutylicum. Appl Microbiol Biotechnol 96:1325-1339. doi: 10.1007/s00253-012-4109-x

Lütke-Eversloh T, Bahl H (2011) Metabolic engineering of Clostridium acetobutylicum: recent advances to improve butanol production. Curr Opin Biotechnol 22:634-647. doi: 10.1016/j.copbio.2011.01.011

Lütke-Eversloh T (2014) Application of new metabolic engineering tools for C. acetobutylicum. Appl Microbiol Biotechnol 98:5823-5837. Doi: 10.1007/s00253-014-5785-5

Madigan M, Martinko J, Dunlap P, Clark D, Brock T (2009) Brock Biology of Microorganisms, 12th edn. Pearson/Benjamin Cummings, San Fransisco

Mao S, Luo Y, Zhang T, Li J, Bao G, Zhu Y, Chen Z, Zhang Y, Li Y, Ma Y (2010) Proteome Reference Map and Comparative Proteomic Analysis between a Wild Type Clostridium acetobutylicum DSM 1731 and its Mutant with Enhanced Butanol Tolerance and Butanol Yield. J Proteome Res 9:3046-3061. doi: 10.1021/pr9012078

McAnulty M, Yen J, Freedman B, Senger R (2012) Genome-scale modeling using flux ratio constraints to enable metabolic engineering of clostridial metabolism in silico. BMC Syst Biol 6:42. doi: 10.1186/1752-0509-6-42 
Millat T, Janssen H, Bahl H, Fischer RJ, Wolkenhauer O (2013a) Integrative modelling of pH-dependent enzyme activity and transcriptomic regulation of the acetone-butanol-ethanol fermentation of Clostridium acetobutylicum in continuous culture. Microbial Biotechnol 6:526-539. doi: 10.1111/1751-7915.12033

Millat T, Janssen H, Thorn G, King J, Bahl H, Fischer RJ, Wolkenhauer O (2013b) A shift in the dominant phenotype governs the $\mathrm{pH}$-induced switch in Clostridium acetobutylicum. Appl Microbiol Biotechnol 97:6451-6466. doi: 10.1007/s00253-013-4860-7

Milne C, Eddy J, Raju R, Ardekani S, Kim PJ, Senger R, Jin YS, Blaschek H, Price N (2011) Metabolic network reconstruction and genome-scale model of butanol-producing strain Clostridium beijerinckii NCIMB 8052. BMC Syst Biol 5:130. doi: 10.1186/1752-0509-5-130

Nölling J, Breton G, Omelchenko M, Makarova K, Zeng Q, Gibson R, Lee H, Dubois J, Qiu D, Hitti J, Production GSC, Finishing, Teams B, Wolf Y, Tatusov R, Sabathe F, Doucette-Stamm L, Soucaille P, Daly M, Bennett G, Koonin E, Smith D (2001) Genome Sequence and Comparative Analysis of the Solvent-Producing Bacterium Clostridium acetobutylicum. J Bacteriol 183:4823-4838. doi: 10.1128/JB.183.16.4823-4838.2001

Papoutsakis E (1984) Equations and calculations for fermentations of butyric acid bacteria. Biotechnol Bioeng 26:174-187. doi: 10.1002/bit.260260210

Petersen D, Welch R, Rudolph F, Bennett G (1991) Molecular cloning of an alcohol (butanol) dehydrogenase gene cluster from Clostridium acetobutylicum ATCC 824. J Bacteriol 173:1831-1834.

Rogers P, Gottschalk G (1993) Biochemistry and regulation of acid and solvent formation in clostridia In: Woods DR (ed) The clostridia and biotechnology, Butterworth-Heinemann, London, pp 25-50

Sauer U, Dürre P (1995) Differential induction of genes related to solvent formation during the shift from acidogenesis to solventogenesis in continuous culture of Clostridium acetobutylicum. FEMS Microbiol Lett 125:115-120. doi: 10.1111/j.1574-6968.1995.tb07344.x

Schaffer S, Isci N, Zickner B, Dürre P (2002) Changes in protein synthesis and identification of proteins specifically induced during solventogenesis in Clostridium acetobutylicum. Electrophoresis 23:110-121. doi: 10.1002/1522-2683(200201)23:1<110::AID-ELPS110>3.0.CO;2-G

Shinto H, Tashiro Y, Yamashita M, Kobayashi G, Sekiguchi T, Hanai T, Kuriya Y, Okamoto M, Sonomoto K (2007) Kinetic modeling and sensitivity analysis of acetone-butanol-ethanol production. J Biotechnol 131:45-56. doi: 10.1016/j.jbiotec.2007.05.005

Straub L (2011) Beyond the Transcripts: What Controls Protein Variation? PLoS Biol 9:e1001146. doi: 10.1371/journal.pbio.1001146

Tummala S, Junne S, Papoutsakis E (2003a) Antisense RNA Downregulation of Coenzyme A Transferase Combined with Alcohol-Aldehyde Dehydrogenase Overexpression Leads to Predominantly Alcohologenic Clostridium acetobutylicum Fermentations. J Bacteriol 185:3644-3653. doi: 10.1128/JB.185.12.3644-3653.2003

Tummala S, Welker N, Papoutsakis E (2003b) Design of Antisense RNA Constructs for Downregulation of the Acetone Formation Pathway of Clostridium acetobutylicum. J Bacteriol 185:1923-1934. doi: 10.1128/JB.185.6.1923-1934.2003

Vasileva D, Janssen H, Hönicke D, Ehrenreich A, Bahl H (2012) Effect of iron limitation and fur gene inactivation on the transcriptional profile of the strict anaerobe Clostridium acetobutylicum. Microbiol 158:1918-1929. doi: 10.1099/mic.0.056978-0

Walter K, Bennett G, Papoutsakis E (1992) Molecular characterization of two Clostridium acetobutylicum ATCC 824 butanol dehydrogenase isozyme genes. J Bacteriol 174:7149-7158.

Welch R, Rudolph F, Papoutsakis E (1989) Purification and characterization of the NADH-dependent butanol dehydrogenase from Clostridium acetobutylicum (ATCC 824). Arch Biochem Biophys 273:309-318.

Wiesenborn D, Rudolph F, Papoutsakis E (1989a) Coenzyme A transferase from Clostridium acetobutylicum ATCC 824 and its role in the uptake of acids. Appl Environ Microbiol 55:323-329.

Wiesenborn D, Rudolph F, Papoutsakis E (1989b) Phosphotransbutyrylase from Clostridium acetobutylicum ATCC 824 and its role in acidogenesis. Appl Environ Microbiol 55:317-322.

Wietzke M, Bahl H (2012) The redox-sensing protein Rex, a transcriptional regulator of solventogenesis in Clostridium acetobutylicum. Appl Microbiol Biotechnol 96:749-761. doi: 10.1007/s00253-012-4112-2

Zhang Y, Hou T, Li B, Liu C, Mu X, Wang H (2014) Acetone-butanol-ethanol production from corn stover pretreated by alkaline twin-screw extrusion pretreatment. Bioprocess Biosyst Eng 37:913-921. doi: 10.1007/s00449-013-1063-7 


\section{Supplementary Material}

\section{Applied Microbiology and Biotechnology}

\section{Coenzyme A-transferase-independent butyrate re-assimilation in Clostridium acetobutylicum - Evidence from a mathematical model}

Thomas Millat $^{1,3}$, Christine Voigt ${ }^{2}$, Holger Janssen ${ }^{2}$, Clare M. Cooksley $^{3}$, Klaus Winzer $^{3}$,

Nigel P. Minton ${ }^{3}$, Hubert Bahl ${ }^{2}$, Ralf-Jörg Fischer ${ }^{2}$, and Olaf Wolkenhauer ${ }^{1,4}$

${ }^{1}$ University of Rostock, Institute of Computer Science,

Department of Systems Biology \& Bioinformatics, Ulmenstr. 69, 18057 Rostock, Germany

${ }^{2}$ University of Rostock, Institute of Biological Sciences, Division of Microbiology, A.-Einstein-Str. 3, 18051 Rostock, Germany

${ }^{3}$ University of Nottingham, School of Life Sciences, BBRSC Sustainable Bioenergy Centre, Clostridia Research Group, Nottingham NG7 2RD, UK

${ }^{4}$ Institute for Advanced Study (STIAS), Wallenberg Research Centre at Stellenbosch University, Stellenbosch 7600, South Africa

Corresponding author: Thomas Millat

Clostridia Research Group,

School of Life Sciences,

University of Nottingham, Nottingham NG7 2RD;

Tel. +44 (0)115 95 15074;

Email: thomas.millat@ nottingham.ac.uk

Table S1 First forward-shift experiment. The time is given in hours and all product concentrations in $\mathrm{mM}$.

\begin{tabular}{cccccccc}
\hline time & $\mathrm{pH}$ & $\mathrm{OD}_{600}$ & acetate & ethanol & acetone & butyrate & butanol \\
\hline 0 & 5.7 & 3.56 & 25.41 & 5.92 & 0 & 22.84 & 0 \\
8 & 5.7 & 7.40 & 37.74 & 8.56 & 0 & 50.47 & 0 \\
18 & 5.7 & 7.16 & 53.39 & 11.75 & 0 & 76.15 & 0 \\
21 & 5.7 & 7.20 & 53.71 & 11.77 & 0 & 75.86 & 0 \\
25 & 5.7 & 6.08 & 55.65 & 12.28 & 0 & 79.05 & 0 \\
31 & 5.7 & 5.97 & 52.12 & 11.25 & 0 & 74.62 & 0 \\
44 & 5.7 & 5.85 & 54.32 & 10.52 & 0 & 84.31 & 0 \\
47 & 5.7 & 5.78 & 53.47 & 9.76 & 0 & 81.99 & 0 \\
52 & 5.7 & 4.68 & 47.59 & 12.47 & 0 & 71.42 & 0 \\
69 & 5.7 & 4.52 & 45.07 & 8.12 & 0 & 66.73 & 0 \\
72 & 5.7 & 4.10 & 42.38 & 7.53 & 0 & 62.45 & 0 \\
79 & 5.7 & 4.30 & 42.17 & 6.56 & 0 & 62.42 & 0 \\
92 & 5.7 & 4.12 & 46.32 & 9.99 & 0 & 61.79 & 0 \\
95 & 5.7 & 4.17 & 46.34 & 9.18 & 0 & 61.46 & 0 \\
101 & 5.7 & 4.00 & 46.67 & 6.02 & 0 & 61.37 & 0 \\
113 & 5.7 & 4.07 & 45.6 & 6.5 & 0 & 59.49 & 0 \\
116 & 5.7 & 4.16 & 43.84 & 6.52 & 0 & 58.46 & 0 \\
116.5 & 5.6 & 4.11 & 44.85 & 6.28 & 0 & 58.8 & 0 \\
117 & 5.5 & 4.17 & 44.87 & 6.22 & 0 & 58.8 & 0 \\
117.5 & 5.4 & 4.22 & 44.78 & 6.38 & 0 & 58.72 & 0 \\
118.5 & 5.3 & 4.29 & 44.79 & 6.16 & 0 & 58.34 & 0 \\
119.5 & 5.2 & 4.25 & 44.39 & 5.3 & 0 & 57.84 & 0 \\
121 & 5.1 & 4.19 & 43.05 & 6.09 & 0 & 55.38 & 0 \\
124 & 4.9 & 3.94 & 39.86 & 4.04 & 0 & 50.44 & 0 \\
127 & 4.8 & 3.73 & 37.52 & 4.16 & 0 & 46.04 & 0 \\
130 & 4.7 & 3.23 & 34.86 & 3.3 & 0 & 40.04 & 0 \\
137 & 4.5 & 2.09 & 27.86 & 2.48 & 0 & 22.46 & 0 \\
141 & 4.5 & 1.84 & 27.32 & 3.24 & 0 & 19.46 & 0 \\
\hline & & & & & & &
\end{tabular}




\begin{tabular}{cccccccc}
\hline time & $\mathrm{pH}$ & $\mathrm{OD}_{600}$ & acetate & ethanol & acetone & butyrate & butanol \\
\hline 145 & 4.5 & 1.72 & 25.62 & 2.63 & 0 & 13.83 & 0 \\
149 & 4.5 & 1.42 & 21.17 & 4.14 & 0 & 14.14 & 14.14 \\
175 & 4.5 & 1.8 & 16.67 & 4.51 & 0 & 11.36 & 11.36 \\
179 & 4.5 & 1.90 & 18.68 & 3.92 & 0 & 12.99 & 12.99 \\
183 & 4.5 & 2.04 & 20.67 & 5.67 & 0 & 8.7 & 12.28 \\
198 & 4.5 & 2.34 & 28.76 & 6.44 & 0 & 4.71 & 23.18 \\
207 & 4.5 & 2.4 & 29.15 & 7.66 & 0 & 2.54 & 24.75 \\
222 & 4.5 & 2.30 & 23.91 & 9.02 & 0 & 2.48 & 20.17 \\
231 & 4.5 & 2.28 & 28.84 & 8.73 & 0 & 3.55 & 24.43 \\
251 & 4.5 & 2.34 & 25.85 & 7.95 & 0 & 5.23 & 22.88 \\
\hline
\end{tabular}

Table S2 Second forward-shift experiment. The time is given in hours and all product concentrations in $\mathrm{mM}$.

\begin{tabular}{cccccccc}
\hline time & $\mathrm{pH}$ & $\mathrm{OD}_{600}$ & acetate & ethanol & acetone & butyrate & butanol \\
\hline 0 & 5.7 & 3.15 & 19.91 & 6.31 & 0 & 22.93 & 0 \\
19 & 5.7 & 4.54 & 41.63 & 7.33 & 0 & 66.94 & 0 \\
23 & 5.7 & 4.23 & 41.74 & 8.82 & 0 & 66.96 & 0 \\
41 & 5.7 & 5.75 & 50.72 & 11.71 & 0 & 87.35 & 1.67 \\
45 & 5.7 & 5.61 & 48.39 & 10.76 & 0 & 79.99 & 2.15 \\
52 & 5.7 & 4.74 & 47.19 & 11.7 & 0 & 79.97 & 2.13 \\
67 & 5.7 & 5.13 & 44.69 & 10.74 & 0 & 75.75 & 1.57 \\
76 & 5.7 & 4.01 & 38.25 & 9.15 & 0 & 64.24 & 0 \\
89 & 5.7 & 3.63 & 37.28 & 9.13 & 0 & 59.9 & 0 \\
100 & 5.7 & 3.67 & 35.87 & 8.88 & 0 & 54.38 & 0 \\
113 & 5.7 & 3.29 & 34.32 & 8.3 & 0 & 50.59 & 0 \\
124 & 4.5 & 3.49 & 35.41 & 6.82 & 0 & 50.65 & 0 \\
137 & 4.5 & 1.89 & 24.07 & 4.66 & 0 & 25.36 & 0 \\
147 & 4.5 & 1.19 & 19.34 & 4.6 & 0 & 10.94 & 0 \\
164 & 4.5 & 2.25 & 20.67 & 5.22 & 0 & 9.5 & 11 \\
190 & 4.5 & 2.12 & 26.24 & 8.44 & 0 & 3.71 & 20.12 \\
208 & 4.5 & 2.37 & 25.13 & 9.2 & 0 & 4.55 & 19.54 \\
219 & 4.5 & 2.04 & 23.64 & 7.99 & 0 & 5.58 & 18.08 \\
232 & 4.5 & 2.13 & 27.56 & 9.05 & 0 & 4.45 & 21.83 \\
243 & 4.5 & 2.48 & 25.92 & 10.11 & 0 & 3.72 & 21.13 \\
258 & 4.5 & 2.42 & 25.93 & 8.74 & 0 & 4.1 & 20.52 \\
\hline
\end{tabular}

Table S3 Third forward-shift experiment. The time is given in hours and all product concentrations in $\mathrm{mM}$.

\begin{tabular}{cccccccc}
\hline time & $\mathrm{pH}$ & OD $_{600}$ & acetate & ethanol & acetone & butyrate & butanol \\
\hline 0 & 5.7 & 6.88 & 40.68 & 10.56 & 0 & 43.59 & 17.38 \\
7 & 5.7 & 7.8 & 56.68 & 12.79 & 0 & 65.27 & 24.12 \\
26 & 5.7 & 5.17 & 50.49 & 11.08 & 0 & 69.19 & 18.73 \\
53 & 5.7 & 3.61 & 47.1 & 7.96 & 0 & 75.29 & 0 \\
72 & 5.7 & 3.54 & 42.57 & 6.76 & 0 & 65.47 & 0 \\
80 & 5.7 & 3.04 & 39.01 & 6.47 & 0 & 58.22 & 0 \\
96 & 5.7 & 3.11 & 35.57 & 5.97 & 0 & 49.71 & 0 \\
103 & 5.7 & 3.12 & 31.41 & 5.43 & 0 & 42.24 & 0 \\
119 & 5.7 & 3.24 & 37.57 & 6.21 & 0 & 53.21 & 0 \\
127.25 & 4.88 & 2.76 & 33.48 & 5.45 & 0 & 45.52 & 0 \\
144 & 4.5 & 1.29 & 18.29 & 3.44 & 0 & 13.66 & 10.04 \\
151 & 4.5 & 1.09 & 17.55 & 3.16 & 0 & 8.13 & 11.67 \\
168 & 4.5 & 1.78 & 18.59 & 3.8 & 0 & 10.42 & 11.89 \\
175 & 4.5 & 1.66 & 18.77 & 3.68 & 0 & 6.75 & 14.44 \\
197 & 4.5 & 1.86 & 21.46 & 5.69 & 0 & 4.95 & 19.51 \\
221 & 4.5 & 1.79 & 18.3 & 4.39 & 0 & 6.57 & 13.94 \\
239 & 4.5 & 1.46 & 14.36 & 3.57 & 0 & 7.08 & 6.26 \\
243 & 4.5 & 1.59 & 13.62 & 3.83 & 0 & 7.15 & 6.78 \\
\hline
\end{tabular}

